\title{
Picking up the mantle of "expert": Assigned roles, assertion of identity, and peer recognition within a programming class
}

\author{
Deborah Fields \\ deborah.fields@usu.edu \\ Emma Eccles Jones College of Education \\ Utah State University \\ 2830 Old Main Hill \\ Logan, UT 84321-2830
}

Noel Enyedy

enyedy@gseis.ucla.edu

Graduate School of Education \& Information Studies

University of California, Los Angeles

2323 Moore Hall

Los Angeles, CA 90095-1521

Keywords: identity, expertise, roles, peer recognition, sociocultural

\begin{abstract}
Changing an established role in a classroom is difficult. It involves constructing a new set of relations within a community. In this paper we investigate how students with newly developed interest and experience programming developed outside the classroom pick up and establish their roles as experts in programming within the classroom community. More specifically, we focus on how two eleven-year-old software designers shifted their established roles in their classroom to gain status as expert programmers. We use an identity lens to understand how peer expertise was established in the context of a classroom community, adopting a multi-facetted perspective of identity by focusing on an individual's narrativization of self, full or peripheral participation amongst a group of people, and individuals' social recognition by others. Our findings point to the importance of both positive positioning by authority figures in the classroom well as activities and roles that provide opportunities to establish intersubjectivity amongst peers in facilitating students' identities as experts in the classroom. Students' willingness to take up a new position in the established activity system also played a role. We consider implications of how making roles flexible within classroom stratification may provide opportunities for more students see themselves as experts.
\end{abstract}

\section{Introduction}

It is widely argued that classrooms can be productively analyzed as communities of practice (Lave \& Wenger, 1991) with shared objectives, routines, norms, tools, and easily recognized 
participation frameworks (Cobb, Wood, Yackel \& McNeal, 1992, Enyedy, Goldberg, \& Welsh, 2005; O'Connor \& Michaels, 1996). One of the most widely recognized features of a typical classroom community is the way that students within the class are stratified and at times stratify themselves (Oakes, 1999). Classrooms are by no means unique in this. Certainly many communities have various ranks, titles or established positions that establish the rights, roles, and responsibilities of participants. However, stratification is particularly pronounced in classrooms and is often identified as a practice that has serious equity implications (McDermott, 1993; Oakes, 1999). For good or ill, a student's position within a classroom's participation framework has implications for how they participate on a day-to-day basis, the degree to which they desire to participate, and their resilience in the face of adversity (Brown, Reveles, \& Kelly, 2005; Hedegaard, 2011; Sfard, 2008). Yet changing an established role is difficult. Practices are just that, routine ways of acting in coordination with others that are repeated or practiced. Taking up a new position or role within a community requires the changing of practices in that community: constructing a new history of relations within the community with the participation and consent of others (Wortham, 2006). Thus the "pipeline" problem so often lamented in STEM education may be in part a participation or identity problem, rather than a purely cognitive or learning problem (Stevens, O'Connor, Garrison, Jocuns, \& Amos, 2008). In computing, the context for this study, Kelleher and Pausch (2005) have argued that these social and cultural barriers are "harder to address than mechanical ones because they are harder to identify and some cannot be addressed through programming systems" (p. 132). One challenge to overcoming these barriers is addressing how to help students who have relevant expertise from outside of a classroom community to build local, social recognition of such expertise in classrooms and to recognize the value of their expertise themselves.

In this paper we investigate how students with newly developed interest and experience in programming developed outside the classroom pick up and establish their roles as experts in programming within the classroom community. More specifically, we focus on how two elevenyear-old software designers shifted their established roles in their classroom to gain status as expert programmers in Scratch (Resnick et al, 2009) amongst their peers. Participation in computer science and entry into technology-related careers by underrepresented groups continues to be low in the United States (NSF, 2006). Further, programming is a particularly opportune disciplinary area to see shifts in participation because of its status in popular culture and folk theories about computers and computer programmers. Much like the beliefs people hold about mathematics - that it is something you are born with an ability to do (Schonefled, 1992; Nasir \& Shah, 2011) - many people believe you either are a computer programmer or you are not. These folk beliefs make the boundary between expert and novice more distinct and harder to cross, and makes those crossings all the more analytically telling.

We use an identity lens to understand how roles as peer experts were newly established in the context of a classroom community as identity reflects both one's self-attributions as well as others' attributions of ability. This approach adopts a multi-facetted perspective by focusing on an individual's narrativization of self (Sfard \& Prusak, 2006; Wortham, 2004), full or peripheral participation amongst a group of people (Lave \& Wenger, 1991), and individuals' social recognition by others (Gee, 2000/1). Our analysis suggests that simple decrees from authority figures such as, "Ask Johnny, he is an expert" do not immediately change either a student's selfattributions or their peers' willingness to recognize their new positions in the classroom structure. Both the individual student and her peers must actively work to establish their positions in the community participation framework. In this process of changing habitual roles 
and/or positions, activities that provide opportunities to establish intersubjectivity about the community's practices, the community's culture, and the participants' relation to the community are critical.

\section{Conceptual framework}

\section{Building an Identity in Practice and Narratives}

Identity as a construct has been theorized and operationalized in a myriad of ways, some quite simplistic and others complicated to the point where it is unclear what isn't identity, but one aspect that is often under-theorized is the relationship between how a person thinks of herself, how others think of her, and how she is constructed in moment-by-moment activity. In this study we draw on a definition of identity as a set of "long-term, living relations between persons and their place and participation in communities of practice" (Lave \& Wenger, 1991, p. 53). This frames identity as a process of social activity enacted in practice with implications for the meaning of the interaction and the organization of future interactions. While we do not adhere to a theory of identity that posits a core sense of self (e.g. Erikson, 1968), we do not wish to ignore the import of a long-term sense of sense of self that some conceptualizations of identity refer to as a narrative (Sfard \& Prusak, 2005) or more recently subjectivities (Sfard, 2008). Indeed, only recently have those who subscribe to a sociocultural definition of identity as built in local social contexts begun to look at the interaction between identities-in-practice as constructed in momentto-moment activity (e.g. Leander, 2002) and narratives of self-understanding (e.g. Sfard \& Prusak, 2005). In this paper we use an approach that considers the relationship within identities as built across events both in social practice, in developing self-narratives (one's selfunderstanding or self-concept), and in others'-narratives (how others think of a person).

In coordinating a practice theory of identity with a "self- and others'-narratives" theory of identity, being an expert involves acting and being received as such in the moment-to-moment flow of activity (practice) as well as thinking about oneself as an expert (self-narrative) and having other people think about oneself as an expert (others'-narratives) outside the flow of that activity. This means that being a programming expert cannot solely rest inside a person's idea of herself. Instead, being an expert must be socially negotiated. Key to both in and out of school activity is the way one positions one's self or is positioned by others with respect to culturally recognized roles, power relationships, and divisions of labor that establish the relevance and meaning of the person's actions within the activity (Ritchie, 2002) or in discursive accounts of that activity at some later time (Vågan, 2011). People use their understandings of what type of activity they are doing and what their role is to limit what they do and say (Philips, 1982, in Erickson \& Mohatt, 1982). Further, what people do and say has direct implications for the meaning that they take away from their experience-meaning that is often retold in narrative form and that can be used to build or forefront new ideas about oneself (Ligorio, 2010).

Below we discuss practice and narrative resources for identity development. Both play a role in the ways that peer expertise is established with the two case studies elaborated in this paper. We are concerned with investigating how two youth who learned to program outside of the classroom (a set of programming practices) became established as peer experts in programming within their classroom, what involved a shift of roles, narratives, and practices.

\section{Moment-to-Moment Resources for Identification}

In interaction positioning can be explicit invocation of a social context and one's role in that context, "I am the teacher here," or positioning can be very subtle. While people do name roles and claim them for themselves or for others, the subtle ways of positioning are perhaps more common and just as effective. Wortham (2008) documents several linguistic expressions and 
strategies used to position people in classroom contexts: what is talked about (reference), how people are evaluated within the participation framework (predication), descriptions of the positions within the participation framework (meta-pragmatic descriptors), the use of insider phrases or jargon to mark membership (evaluative indexicals), and quoting others or reported speech. While not an exhaustive list, these interactional moves typify some of the dialogic events that accumulate to stabilize identities across a local social context like a classroom, "as signs of identity come to presuppose a consistent trajectory of identification that extends across events" (Wortham, 2008, p. 294).

\section{From Moment-to-Moment Resources for Identification to Durable Narratives}

How then are durable narratives built up from moment to moment activity? In the moment-tomoment interaction people take up and provide positions which are resources for regulating one's self and organizing others' activity. These resources and how they are utilized create a very fluid landscape for identity work, but also provide the substrate for a more durable identity narrative. At least two mechanisms are possible to help stabilize and congeal these small-scale phenomena into larger durable phenomena more colloquially recognized as identity. First, interactions can leave material or socially durable traces. For example, in our data group composition is explicitly based on a combination of experts and novices and predisposes students to take up positions in relation to those contrasting roles (Lemke, 2007). Second, one can overtly act in accordance with the trappings of a role, "putting on" an identity and attempting to act it out in ways that others will validate. This is one way that durable narratives can play a role in shaping local identity processes as individuals utilize a socially recognized identity as a "symbolic mediating resource" (p. 211) to mediate their social interactions (Coll \& Falsafi, 2010). This second mechanism is agnostic regarding the degree to which the individual believes the narrative to be true. For example, one can pretend to be brave even when feeling quite scared. From this perspective identity can be interpersonal (between people) before it becomes intrapersonal (Penuel \& Wertsch, 1995). Undoubtedly, this role-playing will at some point require one to dialogically provide resources for recognizable attribution, but the explicit choice to pick up a recognized identity can be a powerful way to create durable, shared interpretations from fleeting interactions.

\section{The Potential for and Process of Identity Development}

Reflecting recent movements to show that identity is not a static 'thing' but rather a dynamic and changing process over time, we use the construct of "trajectories of identification" (Dreier, 2008; Wortham, 2006) to describe youths' developing identities built not only in the classroom where they became established as relative experts in programming but also considering how this was developed across social settings. In this case a "trajectory" is a metaphor for something on a course with direction and momentum, but that may also change due to various forces - in this case personal, social, and cultural influences. Trajectories of identification are shaped by individuals' self-understanding of their life and their future imaginings of self (both incomplete, changing, and cursory), their daily navigation of everyday life, and the ways that local social settings, cultural institutions, and broader cultural values affect and shape them (Dreier, 2008). "Trajectories of identification" imply that people have a past that led up to 'who they are' in the present time and a future that will proceed, in part, from past and present activities. Over "trajectories of events" built across multiple events and multiple time scales (Lemke, 2000), individuals develop reputations as being certain kinds of people in specific social settings with a narrativized past and an imagined future that may change based on future developments, thus the term, trajectories of identification. 
Recent developments of sociocultural research have begun to look at individuals' identity development not only across time but also across spaces. Instead of focusing only on a single trajectory of identification in a class, the focus is shifted to the relationship between multiple trajectories of identification developed in different social settings and how those identities might influence one's behavior and identity development in other settings. Studying the role of movement across settings on individuals' identities sheds light on the challenges of shifting between institutional sets of practices and positionings at home and school (Fleer \& Hedegaard, 2010), the importance of drawing on students' trajectories of identification (Polman, 2010) or Ipositions (Ligorio, 2010) from their lives beyond the classrooms, and the potential for recruiting resources for identity and learning from different sites of participation (Silseth \& Arnesth, 2011, p. 78).

In the context of this paper, we had the privileged position of studying the two youth in multiple settings that characterized their practices of programming differently. They learned to program in an after-school club, and transitioned to programming in a multi-week classroom activity. In this latter situation they were given a public narrative of being "experts" in programming and asked to take up a role of acting as teachers/leaders of their small groups: both the role and the narrative were contested by their peers in small group interactions. Our primary research questions are: how did these two students develop identities as programming experts in the classroom and what resources were brought to bear on these processes of identity development? We use a practice/narrative lens of identity to elaborate how relationships/practices in the classroom shifted to situate the two students as peer experts in programming: being 'named' as such by others, adopting a formal narrative of oneself as an expert, and acting in social interaction.

\section{Context and Methods}

This study is based on a larger connective ethnography (Leander, 2008) of the two focus youth, Tyrone and Lucetta (both pseudonyms), across many social settings of their lives (Fields, 2010). Data collection drew from the three-pronged approach to studying youths' trajectories of identification in social interaction through practice, self-narratives, and others'-narratives, including observations of a preceding after-school Scratch Club, the three-week Scratch Class, and across other subject areas at school and activities at home or with friends (practice); surveys of computing attitudes taken before they learned Scratch in the Club, monthly interviews with the youth (self-narratives), and careful attention to how others talked about the youth (others'narratives). Every meeting of the Scratch Club and Class was recorded by both video-taped and written field note observations, interviews were audio- or video-recorded and transcribed, and visits to homes and other important settings in the two youths' lives were written up in field notes. In addition we also collected their Scratch programs daily throughout the Club and the Class in order to analyze changes in their projects to assess their developing skills as programmers. Though the focus of this paper is on the Scratch Class, analysis of the youths' participation in other settings provided information on how the youth learned Scratch (see Kafai, Fields \& Burke, 2010), how they acted amongst their peers, and their typical kinds of participation in different subjects at school.

The primary setting of this paper includes the two classes of twenty sixth-grade students who participated in using Scratch during a three-week unit in their math class in Spring 2008. Scratch is a media-rich programming language that allows youth to design, share, and remix software programs in the form of games, stories, animations, and art. Scratch (http://scratch.mit.edu) uses a familiar building block command structure (Resnick et al, 2009), 
eliminating thorny debugging processes and the risk of syntax errors. Furthermore, programmed objects can be any imported two-dimensional graphic image, hand-drawn or downloaded from the Web, to further personalize each project. This makes it particularly amenable to an array of novice programmers wanting to build their own software.

In discussions with the teacher, the researchers designed a six-day unit where students made geometric art projects with Scratch in math class to accompany a more general introduction to geometry. Students worked in small groups of two or three sharing a single computer, usually huddling around the screen and taking turns using the mouse. When possible, students with prior experience with Scratch were paired with the students who had no experience with Scratch. The projects involved a cycle of development, revision, and final presentations. For four days, students created the geometric art projects then they uploaded their projects to the Scratch website where students received constructive comments on their projects. On the fifth day students eagerly went online to see their comments and engaged in intense revisions before presenting their projects to the rest of the class at the end of the sixth day.

During the Scratch Class, we videotaped the two small groups in which Tyrone and Lucetta participated, as well as their interactions with other members of Class. Data also include collection of the groups' projects each day and interviews with the small groups at the end of the Class intervention. To analyze the video data itself, we used an iterative approach (Erickson, 2006): going through cycles of examining the corpus of video data, preparing initial hypotheses, and then reviewing the data while adjusting the hypotheses as needed. Specifically, after transcription, we divided videos into short 1-2 minute interactions that illuminated how the student was trying to position herself or how others' actions and reactions shaped the students' ability to take on and maintain a position. Using two-step open coding based on grounded theory (Charmaz, 2000), we coded each chunk for the topic of interaction (e.g. sharing artistic ideas, programming code, problem solving code, off-task conversation), who initiated the interaction (a question, a comment), the type of comment given in response, and the kind of response. We compared these codes to one another and in the second round of coding developed more specific codes related to "procedural" versus "conceptual" questions or suggestions, as these two orientations marked students as insiders or outsiders to the practice of programming in our data. Then we looked at the patterns of these across time with an activity theoretical lens (Cole \& Engeström, 1993) that focused on changes of roles, routines, and tools, and describe the shifts in interactions within the groups in terms of how the small group members treated the local peer experts.

Two categories emerged for the kind of programming and problem solving comments given by Tyrone and Lucetta: "procedural" suggestions and "conceptual" suggestions. "Procedural" suggestions refer to lower-level comments about single pieces of code and their functions such as "move 10 steps," "set pen size to _," or "hide [object]." "Conceptual" suggestions refer to higher-level comments about the relationship between sets of scripts such as identifying a conflict within a set of scripts, identifying a conflict between multiple sets of scripts, and using sophisticated commands to link several scripts or sets of scripts (loops, conditionals, and others). Below we describe how initially there was an underlying conflict between the novices' low-level comprehension of programming that focused on procedural understanding and Tyrone and Lucetta's higher-level comprehension that focused on conceptual understanding of the scripts.

\section{Findings}


Here we describe the ways that Tyrone's and Lucetta's trajectories of identification as programming experts grew within the social context of their sixth-grade classroom, and how this happened through developing practice and brokering explicit self-narratives and others'narratives. We wish to demonstrate how multiple things happened over a series of days that allowed them to overcome the challenges of changing a priori others'-narratives and routines of social interaction. At the same time, this shift in their own roles required a shift of others' roles as their partners had to take up reciprocal roles as novices to acknowledge Tyrone and Lucetta as experts. Below, we briefly describe Lucetta and Tyrone's prior experiences with programming, then illustrate the ways that their peers rejected their relative expertise with programming, how and when the peers shifted to recognizing Lucetta and Tyrone as peer experts and themselves as novices, and the effects of this recognition on the small groups' activities. The path to changing roles in the classroom was not easy. Despite the two youths' recently developed experience in the programming platform used in the classroom (Scratch) and explicit positioning by the teacher as "experts," their peers initially rejected the more valuable expertise that they brought to the projects for several days. This close video analysis of small group interactions demonstrates the challenges the two youth encountered in being recognized in practice as experts among their peers and the contrast in productive collaboration that occurred after their peers realized that the youths' knowledge was valuable to the project creation.

\section{A Brief Background: Tyrone and Lucetta in the After-School Club}

For the purposes of this article, it is important to note that Tyrone and Lucetta's expertise in programming was new and that recognition of this expertise was localized in the after-school club where they had learned it. Neither student had done computer programming before participating in the after-school Club (January - March 2008) that took place mid-year after reputations amongst school peers were already established. Before the Club, they did not think of themselves as very technologically capable as evidenced by their survey responses. Tyrone saw himself as the least technologically proficient of his friends, though he associated himself with a geeky identity. Lucetta saw herself as more proficient than her friends and family, but did not think it was important to her friends that she be good at computers (except for email and chat). Both students were "definitely not" interested in learning to program computers.

During the Club, the two youth learned proficiency with basic programming in Scratch, developing their own styles and types of projects, participating in the online Scratch community, and remixing others' projects (see Author1, 2010b). At the end of the Club we as researchers told them that they were now "junior programmers," providing them with a new narrative for their recent experience. However, to our best knowledge, the social acknowledgement as programmers (or "techies" for that matter) was localized in the Club and did not automatically transfer to recognition in other social settings of their lives. Further, they were not described by themselves or others as "experts;" all Club members had roughly equal expertise in Scratch so it was not a relevant descriptor in that context. As Dreier (2008) points out, changing practices across multiple settings is difficult as it requires many social negotiations. Before the Scratch Class, there appears to have been little instigation for such negotiations, and the youths' identities as having programming expertise were localized to a Club that no longer existed.

\section{A New Activity and New Positionings}

The shift to being situated as experts (and their peers as novices) in programming began when the sixth-grade teacher, Ms. Franklin, introduced a new activity, programming in Scratch, and explicitly framed several students with prior experience in Scratch, including Tyrone and Lucetta, as "experts" in this activity. This created reciprocal roles of novice and expert with an 
opportunity for students who had programming ability to act as experts in the classroom community. With the idea that experts and novices could benefit from working together in groups (i.e., Ching \& Kafai, 2008), the teacher told the students to form groups that mixed novices with an expert (mostly in pairs except for Tyrone who was in a group of 3 ). She also described norms for these roles: experts should act as teachers and leaders by mentoring novices and letting them use the computer while novices were to learn from the experts:

"Some of you are experts, some, novices. Some of you are novices - so there's going to be a huge learning curve. The way people learn best is to actually do, so experts it would behoove you to actually let the novice control the mouse, get to understand. You need to introduce them to all the commands and how the Sprites work. [The researcher] is the expert; I'm the novice, so I'm going to be learning from her."

In this way the teacher elaborated on the positions available to students (a meta-pragmatic descriptor), putting experts in a teaching/leading role and novices in a learning role. These roles reflected the teacher's constructivist philosophy of learning that students would learn by doing; thus the novices needed to "do" while the experts scaffolded their learning. This led to social negotiations within small groups as students figured out how these new roles worked out in practice.

At the same time, Ms. Franklin, a recognized authority in the classroom, also provided an explicit, reified narrative to the experienced students in the title of "expert." This others'narrative was a potential resource for identity development drawn from her knowledge of the students in areas outside of the class (Ligorio, 2010). Yet perhaps because the reciprocal roles themselves were new to the class, the narrative of "expert" by itself it was not enough to change classroom patterns of interactions.

\section{Initial Rejection of Peer Expert and Novice Status}

Though Ms. Franklin had declared to the class that certain members were now experts and others were novices, and that certain interactions should take place (experts encouraging novices to use the computer and learn Scratch commands), it took time for the students to work out these new roles. Meanwhile, a priori social practices between peers continued and the "novice" students paired with Tyrone and Lucetta ignored their higher-level suggestions.

On one level, the social relations within the mixed expertise small groups began along the lines of prior social relations. For instance, Tyrone, known as the meanest kid in sixth grade, tended to ostracize classmates outside of his close group of "geek" friends through his sarcastic sense of humor, especially girls. In his small group his two partners, both girls, rebuffed him and in return he spoke in a negative, sarcastic manner about their mistakes. In fact, the girls treated Tyrone so poorly that Ms. Franklin allowed him to switch groups to work with another pair of girls, Carissa and Diane, who though they treated him a little better still rebuffed most of his suggestions. In contrast, Lucetta had a pre-established role as peacemaker among her classmates. Paired with a friend, Candy, Lucetta put her partner's interests first as Ms. Franklin had directed, making polite suggestions for things that they could do but generally letting her partner lead the direction of the project. Thus upon initial glance, it would appear that Tyrone got along poorly with his partners while Lucetta got along well with hers.

However, after coding procedural and conceptual comments, it became clear that the two experts shared a similar dilemma: whenever they offered higher-level suggestions, whether invited or uninvited, their peers ignored, argued against, or deflected the suggestions. Only procedural answers were solicited and accepted by the novices. In this way both Tyrone and 
Lucetta were treated as experts only peripherally: listened to only when their partners sought basic level knowledge about how to make something move, turn, or change color. Their higher level, algorithmic comments that involved relationships between computer commands were rebuffed.

Consider the following interaction between Lucetta and her partner Candy. Candy asked if they could flutter the cape of their primary sprite, a black man with a pink cape that said "supergirl." Lucetta said this was possible and began to make multiple illustrations of the cape to create an animation of it waving in the wind, but Candy asked to stop before she was finished.

[Second day, 40 minutes into the class, interaction is 2 minutes long.]

Candy: $\quad$ Supergirl should make little-((gesturing with hand))

Candy: $\quad$ Can we make supergirl's cape move?

Lucetta: $\quad$ We just have to change scripts, I mean costumes. So, what should we do.

Candy: $\quad$ But like blowing ((motioning)) in the wind....

((Lucetta works intently with both the mouse and the keyboard.))

Lucetta: $\quad$ Yeah. so::: we just have to. ((clicking on illustration))

All right. Beautiful cape. Make a new one.

Candy: $\quad$ Is there still a regular supergirl one?

Lucetta: $\quad$ Yeah. I copied it.

Lucetta: Wait, this is really skinny, hh... ((jokes follow about supergirl losing weight))

Candy: $\quad$ We can just leave it-it's okay. ((shaking head)) ((Lucetta continues to make adjustments to the script.))

Candy: That's okay- we can just leave it. Let's leave it. In their partnership, Lucetta and Candy followed Ms. Franklin's guidelines in that Candy took up the vision of the project and asked Lucetta for help. However, the partnership broke down in part because Lucetta took over Candy's role at the computer. She started explaining the process on an algorithmic level using some of the insider jargon of Scratch (evaluative indexicals), but also took up the procedural work of illustrating. Candy's statement to "just leave it" was a reassertion of one aspect of her novice role to control the computer but at the same time by refusing to consider Lucetta's knowledge of how to animate a cape she also dismissed putting herself in a novice role. One interpretation is that Candy took up the aspect of the nominated role that was empowering to her (controlling the computer and providing vision for the project) while rejecting the aspects that showed her to need expert help.

This was a recurring pattern of interaction where Candy would say she wanted to quit whenever Lucetta explained or demonstrated a more advanced programming concept. Once after Lucetta said that they needed to "do more steps" to fix a problem, Candy handed over the computer saying, "You fix it - I don't know what you're talking about." However, she only gave Lucetta 30 seconds before becoming impatient and asking to do something different. This pattern culminated on the fourth day when the girls were fixing their project to upload it online. Candy told Lucetta, "This really sucks. It's not working. Do you want to start over? Let's just do something really easy..." In reply, Lucetta expressed that she wanted to continue and that she saw possibilities in fixing the code but in the end allowed Candy to delete everything except for the single image of the supergirl sprite. Here both girls were responsible for not instantiating the new expert-novice roles, though Lucetta did so reluctantly.

Tyrone had similar, if not more obvious rejections of his expertise within his own group. While prior views (others'-narratives) about Tyrone as mean played a role in his partners' 
treatment of him, the difference in knowledge about Scratch also influenced how they responded to Tyrone. While Diane and Carissa asked for and listened to very basic, procedural answers from Tyrone, they either completely ignored him while talking about their visions for the project, ignored the higher-level, conceptual comments that he interjected, or argued directly against such comments. They asked Tyrone about one-step scripts like how to make something "hide," move, or stamp, in contrast with Tyrone's comments that involved relationships between series of scripts, resolving conflicts in the programming scripts or suggesting a new series of scripts that would further his partners' project ideas. Since they did not ask him complex questions, Tyrone's interruptions were attempts to tell his partners algorithmic solutions that they did not recognize needed to be done. Tyrone was willing to take up the role of expert but his partners did not accompany him by becoming novices.

However, this more obvious conflict provided instigation for Ms Franklin to reiterate the narrative of Tyrone as an expert. On the second day, observing that small group relations were tense, Ms. Franklin came over to Tyrone's group to say, "Just checking in, making sure it's balanced and you're being the leader. Good job!" This re-iterated the teacher's narrative about Tyrone as an expert of a certain type (leader). Later that day Tyrone explicitly took up this narrative and internalized it for the first time. Once again having a suggestion rebuffed by his partners, Tyrone vituperously asked, "Why are you looking at me like I'm an idiot? I'm the expert!" This was the first time Tyrone ever referred to himself as an expert in the Class or the Club. Though it had little immediate effect in practice with his partners, it was the beginning of his appropriating an explicit self-narrative as a programming expert.

Thus despite the introduction of a new activity and Tyrone and Lucetta's deeper knowledge of programming, their practices and interactions in the classroom changed little.

\section{Shifts in Identifying Tyrone and Lucetta as Peer Experts}

Starting on day 3 for Tyrone and days $4 / 5$ for Lucetta, the social interactions within the small groups began to change as students shifted positions interactionally as relative experts or novices in programming. Though the transition in Tyrone's group was more obvious, both groups did shift their interactions.

In Tyrone's group, the tension between members built up until the third day when Tyrone once again burst out in verbal frustration. His partners had been debating about how to make a particular effect happen for nearly 15 minutes when Tyrone stood up, interrupted his partners' planning conversation, and pointed to a command on the screen ("hide") that was affecting their other code:

[Third day, 14 minutes into the class, interaction is 2 minutes long]

Tyrone: You're supposed to take "hide"1 out of this.

((Standing and pointing at screen.))

Carissa: $\quad$ No:: We're not going to [inaudible] at the same stupid place.

((Carissa or Diana continue their previous conversation about what they want to happen.))

((30 seconds later Tyrone stands and points at the screen again.))

Tyrone: ((frustrated)): That's a "STA:MP", ! Take "hide" out!

Diana: Oka::y.

Carissa ((annoyed)): Where?

Tyrone ((standing up again to point)): Here! Take "hide" out!

Diana: No, take "stamp" out...

Tyrone ((suddenly calm voice)): Yeah, take "stamp" out too. 
Carissa: 'Cause we don't need this.

Tyrone: Right!

((The girls look at the screen, heads on hands. Tyrone sits back and is quiet.))

In the above conversation, Tyrone pointed to a programming command, "hide," that was interrupting a series of computer commands and preventing the project from working. Carissa rebuffed this attempt. Tyrone tried again half a minute later, his voice rising as he elongated his words and simplified his instruction to removing basic single-level scripts. Finally, a conversation ensued where the partners began to understand each other and Carissa correctly interpreted Tyrone's instruction that there was an unnecessary script. In this shift of interaction, a few things seem to be happening. First, Carissa and Diane seemed to understand more about Scratch - they reached a more algorithmic level of understanding that some commands can interfere with others. Second, Tyrone shifted his comments to a very basic level of taking a command out; his sentences became extremely simple. Perhaps because Carissa and Diane finally had enough experience with Scratch or were engaged enough in solving a particular problem or because Tyrone reached down more to their level, the partners reached intersubjectivity in understanding the other. This became a turning point in the group's collaborative activity. Until this moment Tyrone's attempts to take on the position of expert were in jeopardy precisely because his position was not reciprocated by his peers. Without peer recognition it seems unlikely that Tyrone would develop a durable, narrative identity as an expert, no matter how many attempts he made in that direction.

The turnaround moment for Lucetta and Candy was more subtle, yet there was a shift in the way that Candy treated Lucetta beginning on the fifth day, after they received feedback from the online Scratch community. The suggested idea, namely to have supergirl move and leave flowers wherever he/she went, seemed to provide both girls with renewed energy for working on the project. The interaction below follows the same format as the one described earlier: Candy asked a question, Lucetta provided an answer, and Candy said okay. The main difference is that Candy now used "let's," a plural, instead of handing off all responsibility to Lucetta as she had earlier with, "You fix it."

[Fifth day, 10 minutes into the class, 1 minute interaction]

Candy: ((pointing at screen and gesturing))

Do you think we can make it bouncy?

Lucetta: Don't you think that's like cool when it goes like-, like-

((gesturing at the screen))

Candy: Do you know how to do that?

Lucetta: Yeah.

Candy: Okay well then let's (inaudible).

Since their interactions had not changed significantly before the external feedback, it seems that the recognition from the online Scratch community (an outside authority) enabled Candy to better appreciate that what the Scratch community valued was aligned with what Lucetta was trying to do. It could also be that Candy finally had enough knowledge of Scratch to begin to recognize Lucetta's expertise in practice or that she was willing to accept the role of novice to achieve the cool idea for a project. The short timeline to finishing the project may have influenced this new impetus. While we do not want to place too much significance on the choice of a pronoun, it did seem to signal a shift in their roles where they worked together in a more collaborative fashion, no longer taking over the computer or giving up on certain ideas. 


\section{Changed Practice in Project Design}

After the two groups began to practice roles as experts (Lucetta and Tyrone) and novices (Candy, Carissa and Diane), their interactions continued to change slowly. For instance, though Carissa and Diana verbally acknowledged Tyrone's suggestions, they did not straightaway follow his advice about taking out unnecessary scripts. Still, over the rest of the period they began to implement his advice. They also began to include him in talk about the project, turning their heads to physically include him in their discussions of what they wanted to happen. Tyrone even made them laugh on occasion with his comments, signaling his increasing inclusion in the group. In fact, Tyrone became a better teacher of Scratch along his teacher's educational values: not just commanding his partners, but explaining why things worked, what effects their decisions would have, and what possibilities there could be if they added certain scripts. This change in Tyrone's practices as an expert was possible because of the shifted interactions amongst the groups' members. Where before he was ignored or interrupted, now he could take time to explain things. In these ways Tyrone became a peer expert not only through his teacher's and his own narratives, but also in practice with his peers, resulting in positive effects on their project development.

The relations between Lucetta and Candy also continued to change in ways that situated Lucetta as an expert in programming. The two girls expressed excitement and problem-solved together, with both contributing to the building of the project in ideas and in the construction of scripts, whereas before Candy had organized most of the work, only inviting Lucetta when she was stuck and often ignoring the suggestions Lucetta had made. After the transition, Candy accepted all of Lucetta's suggestions, treating Lucetta as more of an expert and persevering in problem solving over longer periods of time as she accepted Lucetta's recommendations.

Still, the challenge of changing a pre-established role continued to be evident in the ways that class members outside of the two youths' small groups acted toward them. Throughout the Scratch class project, many novice students asked Tyrone and Lucetta for help, but only on a procedural level. After the first two days of the Scratch Class, this practice dwindled, likely because most students understood some basics of Scratch and did not perceive a need for the experts. Still, Tyrone took it upon himself to move about the room and offer unsolicited, conceptual-level advice to various groups. One time he did this, telling one group rather obtusely,

Tyrone: It's obsolete man. ((Looking over the students' shoulders and pointing))

Student: No, it isn't. It's not obsolete.

Tyrone: $\quad$ Some of it - One of the scripts is obsolete.

((Tyrone's friend Eric leans across table to look at the project))

Eric: $\quad$ Yeah I know it's-.

Oh see look, it's supermove, this should say supermove right here.

Student: Is it?

(Pointing to a script))

Though the group began by disbelieving Tyrone, his friend, Eric, intervened to back up and further specify Tyrone's insight. Since Eric had a more established reputation as a computerperson in the class, this action provided further reification of the narrative of Tyrone as a programming expert. Below we discuss the narrative residues left after the Scratch class.

\section{New Narratives of Tyrone and Lucetta as Experts}

The small group interviews after the class show how new narratives about Tyrone and Lucetta as experts had been reified in their own and their classmates' discourse. Reporting their learning, 
Carissa said, "I could listen better..." and that Tyrone helped her and Diane a lot because "he's the expert," reiterating the explicit narrative of Tyrone as an expert in Scratch with the implication that she was a novice by comparison. Tyrone himself said that he had learned "to be a better leader" and "It kinda felt like leadership skills - because I can naturally use the computer," echoing both his teacher's comment that he was "the leader" and a broader cultural narrative about having "natural" skills to use computers. Others in the class also commented about Tyrone's expertise with Scratch, reporting on how he knew how to do things that went beyond the class projects, like making movies and animations in Scratch. Candy, too, spoke articulately about Lucetta's expertise: how it had helped her to learn basic scripts and when to use more nuanced scripts (i.e. "repeat" - a loop command).

I think because Lucetta's really good with Scratch, so I think it helped me to become better with Scratch because I had never done it before.... She kind of taught me how to use the commands, and how to make it work, and how to use the "repeat ${ }^{3}$ " and when not to.

Thus, in the small groups and somewhat beyond, new narratives about Tyrone and Lucetta having expertise in Scratch, parallel to more tacit narratives about the novices learning from them, were formally articulated, based in changed practices yet moving beyond practice to be an explicit idea in many minds. From the changed practices and the newly present narratives, we can argue that Tyrone and Lucetta's roles in their classroom, at least when it came to programming, had changed and that they now had narrative resources or identity positions (Ligorio, 2010) that they could carry to other areas of their lives if they sought to do so.

Tyrone offers us one example of how narratives of expertise in the Scratch class provided him with identity resources elsewhere, namely with his parents. Tyrone leveraged his new selfnarrative as a Scratch programming expert with his parents in his final parent-teacher conference of sixth grade. Previously his parents had expressed concern that his interests in new media and computers, of which they saw Scratch as an example, were detracting from his academic achievement. In his presentation, Tyrone listed Scratch among his three overarching achievements of the year, saying as he looked steadily at his parents, "I became the fifth best Scratch user at REA." Tyrone's statement put him squarely in the company of his four closest "geek" friends (who he saw as the other four "best Scratch users), a contrast to his depiction of himself before the after-school club as only learning from and not teaching his friends and classmates. Later in the presentation he also told his parents that Scratch was computer programming and math, framing it as a legitimate academic activity rather than simply a new media hobby. In these ways Tyrone actively leveraged the narrative of himself as a Scratch expert in a new social setting.

\section{Discussion}

In this paper we have shown how a set of programming practices developed in one social setting, an after-school club, were recruited into a formal narrative by a classroom teacher to frame select students as programming experts in their class. One could argue that a practice in one social setting (club) facilitated a narrative in a new setting (class), which in turn developed new practices and the appropriation of narratives in that setting (class), and that one youth leveraged the narrative in yet another setting (home). Of course the story is more complex than this. As we demonstrated through close video analysis, the teacher's narrative was not the sole factor leading to a shift in roles and narratives in the classroom, shedding greater light on peer pedagogy that has previously taken for granted the higher status of more expert peers due to differing grade levels (Ching \& Kafai, 2008). A complex set of interactions over a period of 
days, influenced by the teacher's narrative, the new activity of programming with Scratch, paired groups with explicit novice/expert roles of a certain sort, and the novices' own learning of and taking up novice roles with Scratch were part of the appropriation of the practices and narratives that shaped Tyrone and Lucetta's identities as programming experts in the class. The two students themselves also willingly took up the mantle of expert in their small group work, rising to the expected role of expert in facilitating the other members' use of Scratch, and growing in this role over the six lessons of the Scratch unit.

It is an exciting phenomenon when two youth take up practices in an academic area (programming) after prior documentation (surveys) that they had been either uninterested or felt a lack of ability in it. Though we would hope that such progress would not stay localized in a specific setting, it often does, as many who run informal workshops (e.g. Hull \& Katz, 2006) or who study the divide between home or hobbies and school (e.g. Leander \& Lovvorn, 2006; Moll, Tapia \& Whitmore, 1993) have found. This paper has illuminated some of the social and cultural barriers (Kelleher \& Pausch, 2005) to moving expertise from one setting into another, among them, shifting sedimented roles in a classroom. Empirically, we have investigated this phenomenon in the context of classrooms and in the development of expert and novice identities in the field of computer programming. However, similar processes of positioning and identity development play out in many other contexts. Parents have notoriously found it difficult to switch from the role of playmate to authority figure with their children. Professionals may struggle to inhabit a new position within the workplace. It is likely that these difficulties arise in part from the fact that identity and one's position in a social system are joint accomplishments of the participants. Wishing, or in this case asserting an identity, does not necessarily make it so as others have to accept you in that position and may even need to take up complementary roles, but as our data have shown, picking up or asserting an identity is often a good place to start.

First, and perhaps not surprisingly, our analysis showed that new classroom activities create opportunities to develop one's own relation to the larger activity of schooling in new ways as one negotiates and defines one's role in the new practice. Our analysis also showed, however, that one's new relationship is not made new from whole cloth but is constrained and borrows from existing practices and positions within the community; therefore new activities also offer ways to calcify existing relations. Initially it was unclear whether Tyrone would be able to move from his marginalized position in the classroom to take up the mantle of programming expert. The teacher played a key role in narrativizing Tyrone and Lucetta as experts in programming and setting up small groups with roles (expert/novice) that reified that narrative. In this way the teacher served as a broker (Wenger, 1998), sharing her knowledge of the students' capabilities from the club (which she knew about from a relationship with the authors) and introducing a new potential identity for them. However, this was not enough. One may be positioned easily enough, but staying there and incorporating that position into one's narrative identity is a joint accomplishment of the community.

In particular, this study sheds light on how youth had to take up respective roles as experts and novices within their small groups. Lucetta and Tyrone could not well be positioned as experts in practice in their small groups until their partners took up more novice roles in practice, something that appeared to take both new learning of programming by the novices and achieving dialogic intersubjectivity amidst the groups. Early on, Carissa, Diane, and Candy were focused on procedural level programming and asserted the pre-existing relationships of the classroom that were still available to them, in essence refusing to take up roles as novices. It is impossible from our analysis to say why they were unwilling to take up such roles, but one 
reason is likely that "novice" roles positioned them with less power in the small group. However, when the groups came to a common understanding of what was possible in Scratch and began talking about those things in the same way, we saw a positive shift in how the group-mates treated their experts. One interpretation of this is that Candy, Carissa, and Diane learned enough about Scratch to realize what would be possible with "expert" help. In other words, they were willing to take up novice roles when the gain from those roles (having a project that worked in a way they desired) was greater than the loss of power. To further understand the interactions between motivations and learning in taking up novice and expert roles, future research should have an explicit focus on both novice and expert positions in small group activities.

Through this negotiation students were able to upend the existing set of relations within the classroom activity system. Students who were typically not at the top of the classroom stratification were able negotiate new positive positions and identities as experts while other students subsequently took up positions as novices. However, we would be remiss if we failed to point out that this does little to disrupt the general system of stratification. It only disrupts who is where, and only to the degree that the participants seek out change. Still, introducing more flexibility in how students are positioned relative to one another is a positive accomplishment for at least two reasons. First, it highlights for students that they can have multiple academic identities, rather than one monolithic school identity. To the degree that positions within the classroom are seen as flexible and many people can become experts in different contexts, and to the degree that these contexts are all seen as valuable, this seems to mitigate some of the equity concerns associated with the way classrooms currently stratify their students. Second, it opens up the possibility of new practices, tools, and divisions of labor that develop hand-in-hand with the new set of relations between people. For example, in this class a division of labor emerged where experts were the conceptual leaders but were not in control of implementing their own ideas.

Finally, our analysis shows that identity change takes time and multiple resources to take up one's new position. In our data, people's willingness to repeatedly take up their position in the activity system was related to the value they saw in that position and hints at broader cultural narratives that likely played a role in taking up the mantles of programming expert or novice. For Tyrone, being an expert in programming helped him outside of the classroom to establish himself with the peer group he wanted, allowed him to successfully show his parents that his interests in computers and video games had some relevance to their academic goals for him, and provided entry into a cultural narrative about "naturally" being good with computers. On the other hand, Lucetta let the idea that she was an expert sit quietly in the background rather than leveraging that narrative resource outright. One reason for this was likely the idea that she already thought of herself as more proficient in using computers than her family and friends, and her friends seemed to care more about social uses of computing (email and chat) than creating with computers. She (and her friends) may also have been influenced by a prevalent cultural narrative of computing as a masculine domain that has negatively affected many girls' identity development in computing (Brickhouse \& Potter, 2001; Stepulvage, 1999). This points to the need for further research into the complexity of motivations and interests behind youths' appropriation of narratives and the ways that practices and relationships in multiple settings as well as cultural narratives about gender and natural ability affect their appropriation of identities.

There are a number of limitations to this research. We have analyzed the development of two youth across settings, highlighting their development as experts over a relatively short period of time in a three-week, six-lesson class. Though our data extends across six months and many settings, it does not allow us to see how students progressed in their identities as experts in 
programming after the school year. We did present the findings to both youth several months after the school year and they verified our interpretations of their experiences. Future studies could take a more extended look at both youth positioned as novices and as experts in different classroom domains and how those identities may extend (or not) across settings. Studying not just individual youths' identities but also group identities and degrees of ownership in a collaborative product would also have research value.

In this paper we have demonstrated that while changing roles in a classroom is difficult and complex, the rewards hold great potential for supporting students' identity development as experts in a specific area, namely through their embracing of narratives as experts and their pursuit of related academic practices. It is one way to help students link relevant expertise from outside to inside a classroom community. Our findings point to the importance of both positive positioning by authority figures in the classroom (e.g. the teacher) as well as activities and roles that provide opportunities to establish intersubjectivity and reciprocal roles amongst peers. Students' willingness to take up a new position in the established activity system also played a role in the degree to which they took up an enduring narrative of expertise about themselves. Perhaps most importantly, while classroom stratification has many documented negative effects on students, our study suggests that making it flexible may provide more students with opportunities to see themselves as experts. In other words, developing flexibility in students' positioning relative to one another (i.e. flexible roles that may shift depending on subject area or curricular unit) may be a positive accomplishment in regard to overcoming barriers to connecting expertise across settings. Further research is needed to document the ramifications of this over time and across school disciplines.

\section{Endnotes}

(1) "Hide" is a script in Scratch that causes a sprite (object) to disappear from view. If one wants an object to appear sometimes after the program is started, then one must put "hide [sprite]" at the beginning of the project and "show [object]" when one wants it to appear. The "hide" command that Tyrone pointed to was probably misplaced and caused the sprite to be invisible.

(2) "Stamp" is a script that causes the sprite to stamp its image onto the screen.

(3) "Repeat" is one kind of loop in Scratch, allowing a set of scripts to be repeated for a set number of times.

\section{Acknowledgements}

The work reported in this paper was supported by a UCLA mentorship fellowship to the first author and a grant from the National Science Foundation (NSF-0325828) to Yasmin Kafai. A prior version of this paper was presented at the 2011 Computer Supported Collaborative Learning conference. We wish to thank Andy Blunden and three blind reviewers for their helpful comments on earlier drafts of this article. In addition we thank Yasmin Kafai for her prior readings and feedback on the paper and William Q. Burke for his assistance with data reduction.

\section{References}

Brickhouse, N. W., \& Potter, J. T. (2001). Young women's scientific identity formation in an urban context. Journal of Research in Science Teaching, 38, 965-980.

Brown, B.; Reveles, J.; \& Kelly, G. (2005) Scientific literacy and discursive identity: A theoretical framework for understanding science education. Science Education, 89, 779802. 
Charmaz, K. (2000). Grounded theory: objectivist and constructivist methods. In N. K. Denzin, \& Y. S. Lincoln (Eds.), Handbook of qualitative research (pp. 509-535). Thousand Oaks, CA: Sage.

Ching, C. C., \& Kafai, Y. B. (2008). Peer pedagogy: student collaboration and reflection in a learning through design project. Teachers College Record, 110 (12), 2601-2632.

Cobb, P., Wood, T., Yackel, E. \& McNeal, B. (1992). Characteristics of classroom mathematics traditions: An interactional analysis. American Educational Research Journal, 29(3), 573-604.

Cole, M. \& Engeström, Y. (1993). A cultural-historical approach to distributed cognition. In Salomon, G., \& Brown, J. S. (Eds.), Distributed cognitions: Psychological and educational considerations. Cambridge: Cambridge University Press, 1-46.

Coll, L. \& Falsafi, L. (2010). Learner identity. An educational and analytical tool. Revista de Educación, 353(1), 211-233.

Dreier, O. (2008). Psychotherapy in everyday life. Cambridge: Cambridge University Press.

Enyedy, N., Goldberg, J., \& Welsh, K. M. (2005). Complex dilemmas of identity and practice. Science Education 90(1) 68-93.

Erickson, F. (2006). Definition and analysis of data from videotape: Some research procedures and their rationales. In J. Green, Camilli, G., \& Elmore, P. (Ed.), Handbook of complementary methods in educational research. (3rd ed.) American Educational Research Association.

Erickson, F. \& Mohatt, C. (1982). Cultural organization and participation structures in two classrooms of Indian students. In G. Spindler, (Ed.), Doing the ethnography of schooling (pp. 131-174). New York: Hold, Rinehart \& Winston.

Erikson, E. H. (1968) Identity: Youth and crisis. Norton: New York.

Fields, D. A. (2010). Trajectories of identification across social spaces: Intersections between home, school, and everyday settings. Unpublished dissertation. University of California, Los Angeles.

Fleer, M. \& Hedegaard, M. (2010). Children's Development as Participation in Everyday Practices across Different Institutions. Mind, Culture, and Activity, 17, 149-168.

Gee, J. P. (2000/1). Identity as an analytic lens for research in education. Review of Research in Education, 25, 99-125.

Hedegaard, M. (2011). A cultural-historical approach to children's development of multiple cultural identities. In M. Kontopodis, C. Wulf, \& B. Fichtner (Eds), Children, development and education: Cultural, historical, anthropological perspectives, 117-135.

Hull, G. A. \& Katz, M.-L. (2006). Crafting an agentive self: Case studies of digital storytelling. Research in the Teaching of English, 41(1), 43-81.

Kafai, Y. B., Fields, D. A., \& Burke, W. Q. (2010). Entering the clubhouse: Case studies of young programmers joining the online Scratch communities. Journal of Organizational and End-User Computing, 22(2), 21-35.

Kelleher, C. \& Pausch, R. (2005). Lowering the barriers to programming: A taxonomy of programming environments and languages for novice programmers. ACM Computing Surveys, 37(2), 83-137.

Lave, J. \& Wenger, E. (1991). Situated learning and legitimate peripheral participation. Cambridge: Cambridge University Press.

Leander, K. M. (2002). Locating LaTanya: The situated production of identity artifacts in classroom interaction. Research in the Teaching of English, 37, 198-250. 
Leander, K. M. (2008). Toward a connective ethnography of online/offline literacy networks. In D. Leu, J. Cairo, M. Knobel, \& C. Lankshear (Eds.) Handbook of research on new literacies. New York: Erlbaum, 33-65.

Leander, K. M., \& Lovvorn, J. F. (2006). Literacy networks: Following the circulation of texts, bodies, and objects in the schooling and online gaming of one youth. Cognition and Instruction, 24(3), 291-340.

Lemke, J. L. (2007). Video epistemology in- and outside the box: Traversing attentional spaces. In R. Goldman, S. Barron, S. Derry and R. Pea (Eds.) Video Research In The Learning Sciences, Mahwah, NJ: LEA, 37-51.

Lemke, J. L. (2000). Across the Scales of Time: Artifacts, Activities, and Meanings in Ecosocial Systems, Mind, Culture, and Activity, 7(4), 273-290.

Ligoro, M. B. (2010). Dialogical relationship between identity and learning. Culture \& Psychology, 16, 93-107.

McDermott, R. (1993). The acquisition of a child by a learning disability. In S. Chaiklin \& J. Lave (Eds.), Understanding practice (pp.269-305). New York: Cambridge University Press.

Moll, L. C., Tapia, J. \& Whitmore, K. F. (1993). Living knowledge: The social distribution of cultural resources for thinking. In Salomon, G., \& Brown, J. S. (Eds.), Distributed cognitions: Psychological and educational considerations. Cambridge: Cambridge University Press, 139-163.

Nasir, N. S. \& Shah, N. (2011). On defense: African American males making sense of racialized narratives in mathematics education. Journal of African American Males in Education, 2(1), 24-45.

National Science Foundation, Division of Science Resources Statistics (2010). Science and Engineering Degrees, by Race/Ethnicity of Recipients: 1997-2006. Detailed Statistical Tables NSF 10-300. Arlington, VA: Author. Retrieved January 6, 2012 from http://www.nsf.gov/statistics/nsf10300/

Oakes, J. \& Lipton, M. (1999). Teaching to change the world. Boston: McGraw Hill.

O'Connor, M. C.,\& Michaels, S. (1996). Shifting participant frameworks: Orchestrating thinking practices in group discussion. In D. Hicks (Ed.), Discourse, learning, and schooling (pp. 63-103). New York: Cambridge University Press.

Penuel, P. R. \& Wertsch, J. V. (1995). Vygotsky and identity formation: A sociocultural approach. Educational Psychologist, 30(2), 83-92.

Philips, S. (1982). The Invisible Culture: Communication in Classroom and Community on the Warmsprings Indian Reservation. New York: Longman.

Polman, J. L. (2010). The zone of proximal identity development in apprenticeship learning. Revista de Educación, 353(1), 129-155.

Resnick, M., Maloney, J., Monroy- Hernández, A., Rusk, N., Eastmond, E., Brennan, K., Millner, A., Rosenbaum, E., Silver, J., Silverman, B., Kafai, Y. B. (2009). Scratch: Programming for All. Communications of the ACM, November, 2009.

Ritchie, S. M. (2002). Student positioning within groups during science activities. Research in Science Education, 32(1), 35-54.

Schoenfeld, A (1992). Learning to think mathematically: Problem solving, Metacogntition, and Sensemaking in Mathematics. In D. Grows (Ed.) Handbook for research on mathematics teaching and learning, (pp. 334-370). New York, NY: Macmillian.

Sfard, A. (2008). Thinking as communicating: Human development, the growth of discourses, 
and mathematizing. New York, NY: Cambridge University Press.

Sfard, A., \& Prusak, A. (2005). Telling identities: In search of an analytic tool for investigating learning as a culturally shaped activity. Educational Researcher, 34(4), 14-22.

Silseth, K. \& Arnseth, H. C. (2011). Learning and identity construction across sites: A dialogical approach to analysing the construction of learning selves. Culture \& Psychology, 17, 6580.

Stepulevage, L. (1999). Becoming a technologist: Days in a girl's life. Information, Communication \& Society, 2(4), 399-418.

Stevens, R., O'Connor, K., Garrison, L., Jocuns, A., \& Amos, D. M. (2008). Becoming and engineer: Toward a three dimensional view of engineering learning. Journal of Engineering Education, 97(3,)355-368.

Wenger, E. (1998). Communities of practice: Learning, meaning, and identity. Cambridge: Cambridge University Press.

Wortham, S. (2004). From good student to outcast: The emergence of a classroom identity. Ethos, 32(2), 164-187.

Wortham, S. (2006). Learning identity: The joint emergence of social identification and academic learning. Cambridge: Cambridge University Press.

Wortham, S. (2008). "The objectification of identity across events." Linguistics \& Education, 19(3). p. 294-311.

Vågan, A. (2011). Towards a sociocultural perspective on identity formation in education. Mind, Culture, and Activity, 18, 43-57. 